\title{
AUTOMORPHISM OF A FINITE GROUP SCALAR ON THE COSETS OF A SUBGROUP
}

\author{
SAID SIDKI
}

\begin{abstract}
Let $G$ be a finite group, $\sigma$ an automorphism of $G$, $M$ a $\sigma$-invariant subgroup of $G$, and $n$ a fixed integer. If $\sigma(g) \in g^{n} M$ for all $g \in G$ then there exists a $\sigma$-invariant normal subgroup $K$ of $G$, contained in $M$, with $\sigma(g) \in g^{n} K$ for all $g \in G$.
\end{abstract}

1. Introduction. The purpose of this paper is to prove the following result.

THEOREM. Let $G$ be a finite group, $\sigma$ an automorphism of $G, M a$ ainvariant subgroup of $G$, and $n$ a fixed integer. If $\sigma(g) \in g^{n} M$ for all $g \in G$ then there exists $K$ a $\sigma$-invariant subgroup of $M, K \triangleleft G$, and $\sigma(g) \in g^{n} K$ for all $g \in G$.

It follows from the theorem that $\bar{G}=G / K$ is $n$-abelian; i.e., $(a b)^{n}=a^{n} b^{n}$ for all $a, b \in G$. Such groups have been classified by Alperin in [1].

It is well known that for $n=1, K$ can be taken to be $\langle[G, \sigma]\rangle$.

When $M$ is the trivial subgroup, we refer to $\sigma$ as a scalar automorphism. In this case $[G, \sigma] \subseteq Z(G)$, and $\sigma$ is a fixed point free automorphism iff $(n-1, o(G))=1($ see $[2])$.

Throughout this paper $G$ will stand for a finite group and $\sigma$ for an automorphism of $G$. We will find it convenient to regard $G$ and $\langle\sigma\rangle$ as embedded in the semidirect product of $G$ by $\langle\sigma\rangle$; e.g., $[g, \sigma]=g^{-1} \sigma^{-1} g \sigma$. The rest of our notation is standard (see [4] or [5]).

\section{Preliminary results.}

LEMMA 1. Let $\sigma$ be an automorphism of $G$ of prime order $p$. Suppose $[x, \sigma, \sigma]=e$ for all $x \in G$ such that $x^{p}=e$. Suppose furthermore that for some $y \in G,[y, \sigma, \sigma]=e$, yet $[y, \sigma] \neq e$. Then $O_{p}(G) \neq E$.

Proof. Denote $C_{G}(\sigma)$ by $H$. Let $y \in G$ such that $e \neq z=[y, \sigma] \in H$. Then $z^{p}=e, z_{1}=\left[y^{-1}, \sigma\right]^{-1}=[y, \sigma]^{y^{-1}}$, and $z_{1} \in H$.

Let $t \in H, t^{p}=e$. Then, as $\left[t^{y}, \sigma, \sigma\right]=e$, we have, from $e=\left[t^{y}, \sigma \sigma^{-1}\right]=$ $\left[t^{y}, \sigma^{-1}\right]\left[t^{y}, \sigma\right]$

$$
\left[t^{y}, \sigma\right]^{-1}=\left[t^{y}, \sigma^{-1}\right]
$$

Received by the editors May 17, 1971 .

AMS 1970 subject classifications. Primary 20D45, 20F55; Secondary 20D05.

Key words and phrases. Scalar automorphism, fixed point free automorphism, Engel element, Baer's theorem, Odd Order paper, involution, simple group.

(c) American Mathematical Society 1972 
Since $t^{y z}=t^{y}\left[t^{y}, \sigma\right]$

$$
\left[t^{y z}, \sigma^{-1}\right]=\left[t^{y}, \sigma\right]^{-1}
$$

and so $\left[t^{y}, \sigma\right]$ commutes with $z$. Thus, $z_{1}$ commutes with $\left[t, z_{1}\right]$. Let $t=z_{1}^{-h}$, $h \in H$. Then the iterated commutator $\left[h, 3 z_{1}\right]=e$. In other words, $z_{1}$ is a left Engel element of $H$ and so by a theorem of Baer [5, p. 212], $z_{1} \in O_{p}(H)$. If $y^{-1}$ is substituted for $y$ in the preceding argument we get $z \in O_{p}(H)$.

Let $v \in Z\left(O_{p}(H)\right), o(v)=p$. If $x \in G$ and $o(x)=p$, then $v^{x} \in H$. Let $y=v^{-g}$, $g \in G$. Then, $[y, v]=\left[v^{-g}, v\right]=[g, 2 v]^{v^{-1}}$ is an element of $H$. Since $v \in O_{p}(H)$, $[g, k v]=e$ for some positive integer $k$. Thus $v$ is a left Engel element of $G$; hence, $v \in O_{p}(G) \neq E$.

Definition AND Remark. Let $n$ be a fixed integer and $G$ a finite group. Define $\mathscr{N}(G)=\langle g| g \in G$ and $\left.g^{n}=e\right\rangle . \mathscr{N}(G)$ is a characteristic subgroup of $G$. Let $\mathscr{N}_{0}=\mathscr{N}(G)$ and define $\mathscr{N}_{i}$ inductively as the preimage of $\mathscr{N}\left(G / \mathscr{N}_{i-1}\right)$ in $G$. Let $\mathscr{N}_{\infty}=\bigcup_{i=0}^{\infty} \mathscr{N}_{i}$. Then $o\left(G / \mathscr{N}_{\infty}\right)$ and $n$ are coprime.

In the sequel, we will let $n$ be a fixed integer, and $M$ a $\sigma$-invariant subgroup of $G$ such that $\sigma(g)=g^{n} m(g), m(g) \in M$, for all $g \in G$.

We begin with an inheritance-type lemma the proof of which is direct.

LEMma 2. Let $F$ and $H$ be $\sigma$-invariant subgroups of $G, F \triangleleft H$, and $\bar{\sigma}$ the automorphism induced by $\sigma$ on $\bar{H}=H / F$. Then

is $\bar{\sigma}$-invariant and

$$
\overline{M \cap H}=(M \cap H) F / F
$$

for all $\bar{h} \in \bar{H}$.

$$
\bar{\sigma}(\bar{h})=\bar{h}^{n} \overline{m(h)}, \quad \overline{m(h)} \in \overline{M \cap H}
$$

LEMMA 3. $[M, \sigma]^{G} \mathscr{N}_{\infty} \subseteq M$.

Proof. Let $g \in G, t \in M$.

$$
\begin{aligned}
\sigma(g) & =g^{n} m(g), \\
\sigma\left(t^{-1} g t\right) & =t^{-1} g^{n} t m\left(g^{t}\right)=\sigma(t)^{-1} \sigma(g) \sigma(t)=\sigma(t)^{-1} g^{n} m(g) \sigma(t) .
\end{aligned}
$$

Thus, $\left[g^{n}, \sigma(t) t^{-1}\right] \in M$. If $n$ and $o(G)$ are coprime then $[G,[\sigma, t]] \subseteq M$ for all $t \in M$; thence, $[\sigma, M]^{G} \subseteq M$. Now if $\mathscr{N}_{\infty} \subseteq M$, then by Lemma 2 we may translate the situation to $G / \mathscr{N}_{\infty}$; in which case we are finished. We may assume that there exists $g \in G, g \neq e$ and $g^{n}=e$. Then $\sigma(g) \in g^{n} M=M$ and so $g \in M$; i.e., $\mathscr{N}_{0} \subseteq M$. On applying induction to $o(G)$, and since $o\left(G / \mathscr{N}_{0}\right)<$ $o(G), \mathscr{N}_{\infty} \subseteq M$; the proof is finished.

Lemma 4. Suppose $\left.\sigma\right|_{M}=1$. Then

(i) $G^{n^{0(\sigma)}-1} \subseteq M$,

(ii) $g^{n-1}$ commutes with $m(g)$ for all $g \in G$,

(iii) for any subgroup $H$ of $G,\langle[H, \sigma]\rangle$ is $\sigma$-invariant. 
Proof. Part (i) follows from $\sigma^{i}(g) \in g^{n^{i}} M, i$ integer $\geqq 0$. As for part (ii), $\sigma\left(g^{-1}\right)=g^{-n} m\left(g^{-1}\right)=m(g)^{-1} g^{-n}$ implies $g^{n} m(g) g^{-n}=m\left(g^{-1}\right)^{-1}$. On expanding each term of $\sigma(g)=\sigma\left(g^{1-1 / n}\right) \sigma\left(g^{1 / n}\right)$ and employing the previous equation we get $a=m(g)^{g^{-1}} \in M$; now (ii) follows from $\sigma(a)=a$. To prove part (iii) it suffices to observe, $[g, 2 \sigma]=\left[g^{n-1}, \sigma\right]$.

Lemma 5. Suppose $o(\sigma)=p, p \in \pi(G)$. Let $n^{p} \equiv 1$ modulo $\exp (G)$, and $[M, \sigma]=E$. Then $[G, \sigma] \subseteq N_{G}(P)$, where $P \in \operatorname{Syl}_{p}(G)$.

Proof. We proceed by induction on $o(G)$. We may assume that for some $x \in G$ such that $o(x)=p, x \notin M$. Then, as $n \equiv 1 \bmod p$, we get $[x, 2 \sigma]=e$ and $[x, \sigma] \neq e$. Thus by Lemma $1, O_{p}(G) \neq E$. The conclusion follows by applying the inductive hypothesis to $G / O_{p}(G)$.

3. Proof of the theorem. Let $G$ be a counterexample of minimal order. Clearly, the only subgroup of $M$ normal in $G$ is $E$. From Lemmas 3 and 4 we conclude that $[M, \sigma]=E,(n, o(G))=1, n^{o(\sigma)} \equiv 1$ modulo $\exp (G)$. In addition, we may regard $M$ to be a maximal subgroup of $G$. There are proper nontrivial normal subgroups in $G$. This is certainly true if $(o(\sigma)$, $o(G)) \neq 1$, as is implied by Lemma 5. On the other hand if $o(\sigma)$ and $o(G)$ are coprime then $o(G)$ is odd; otherwise, if $x \in G$ and $o(x)=2$, then $x^{n}=x$, $[x, \sigma]^{o(\sigma)}=e$, and $x \in M$. In this case, by the Odd Order paper [3], $G$ is solvable.

Let $H$ be a minimal nontrivial normal subgroup in $G$; therefore $H$ is semisimple and $H \neq G$. Then $G=H M$ and $R=\langle[H, \sigma]\rangle=\langle[G, \sigma]\rangle$ is $\sigma-$ invariant nontrivial normal in $G$. So, $G=R M$.

Suppose $G \neq R$ and let $M_{0}=R \cap M$. Furthermore, suppose $M_{0}$ is trivial. Then $\sigma$ is fixed point free on $R$ and is scalar $n$ on $R$. Hence $R$ is an elementary abelian $q$-group for some prime $q$. Let $x \in R, t \in M$. Then $[x t, \sigma]=$ $(x t)^{n-1} m(x t)$, and $[x t, \sigma]=[x, \sigma]^{t}=\left(x^{n-1}\right)^{t}$. Since $(x t)^{n-1}=f(x) t^{n-1}$ where $f(x) \in R$, we conclude $t^{n-1} m(x t)=e$ and $f(x)=\left(x^{n-1}\right)^{t}$; so by Lemma 4, $t^{n-1}$ commutes with $\left(x^{n-1}\right)^{t}$. Moreover, since $n-1$ and $o(x)$ are coprime, $x$ commutes with $t^{n-1}$. Hence, $R$ commutes with $M^{n-1}$; so, $M^{n-1}$ is trivial. Furthermore, from $t^{n-1}=m(x t)^{-1}=e$, we get $\sigma(x t)=(x t)^{n}$; therefore, $R=[G, \sigma] \subseteq Z(G)$, and so $M \triangleleft G$ which is impossible.

Hence, $E \neq M_{0} \triangleleft M$ and $\sigma(k) \in k^{n} M_{0}$ for all $k \in R$. Since $R \neq G$, there exists $K_{0}$ a subgroup of $M_{0}, K_{0} \neq E, K_{0} \triangleleft R$, such that $\sigma$ induces a scalar $n$ on $R / K_{0} . K_{1}=K_{0}^{M} \subseteq M_{0}, K_{1} \triangleleft R$ and so $K_{1} \triangleleft G$ which is impossible.

So far then, $G=R=H \sigma(H), H \cap \sigma(H)=E$, elements of $H$ commute with those of $\sigma(H)$, and $H$ is simple nonabelian. Again we make use of the Odd Order paper and let $x$ be an involution in $H$. Since $x$ commutes with $[x, \sigma],[x, \sigma]^{2}=e$. Also, since $[x, \sigma] \in M, H$ is $\sigma^{2}$-invariant and $\sigma^{2}$ is trivial on $H$. Consequently, $o(\sigma)=2$; however, using Lemma $5, R \neq G$. A contradiction is reached and the theorem is established. 
ACKnOwledgement. I wish to thank Professor G. Glauberman for his advice and encouragement. My thanks are also due to the University of Chicago for providing me with the opportunity to visit and study there in 1970-71.

\section{REFERENCES}

1. J. L. Alperin, A classification of n-abelian groups, Canad. J. Math. 21 (1969), 1238-1244. MR 40 \#1458.

2. C. D. H. Cooper, Power automorphisms of a group, Math. Z. 107 (1968), 335-356. MR 38 \#4550.

3. W. Feit and J. G. Thompson, Solvability of groups of odd order, Pacific J. Math. 13 (1963), 775-1029. MR 29 \#3538.

4. D. Gorenstein, Finite groups, Harper and Row, New York, 1968. MR 38 \#229.

5. E. Schenkman, Group theory, Van Nostrand, Princeton, N.J., 1965. MR 33 \#5702.

Department of Mathematics, Universidad de Oriente, Cumaná, Venezuela

Department of Mathematics, University of Chicago, Chicago, Illinois 60637

Current address: Department of Mathematics, Universidade de Brasília, Brasilia-D. F.-Brazil 